\title{
Machine learning algorithms for electrical appliances monitoring system using open-source systems
}

\author{
Viet Hoang Duong, Nam Hoang Nguyen \\ Department of Instrumentation and Industrial Information, Hanoi University of Science and Technology, Vietnam
}

\section{Article Info}

Article history:

Received May 9, 2021

Revised Dec 20, 2021

Accepted Dec 30, 2021

\section{Keywords:}

Electrical appliance states

Machine learning

Open-source

Saving energy

ABSTRACT

Two main methods to minimize the impact of electricity generation on the environment are to exploit clean fuel resources and use electricity more effectively. In this paper, we aim to change the user's electricity usage by providing feedback about the electrical energy consumed by each device. The authors introduced two devices, load monitoring device (LMD) and activity monitoring device (AMD). The function of the LMD is to provide feedback on the operating status and energy consumption of electrical appliances in a home, which will help people consume electrical energy more efficiently. The parameters of LMD are used to predict the on/off state of each electrical appliance thanks to machine learning algorithms. AMD with audio sensors can assist LMD to distinguish electrical devices with the same or varying power over time. The system was tested for three weeks and achieved a state prediction accuracy of $93.60 \%$.
\end{abstract}

This is an open access article under the CC BY-SA license.

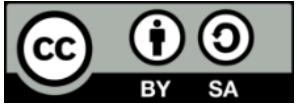

\section{Corresponding Author:}

Nam Hoang Nguyen

School of Electrical Engineering, Hanoi University of Science and Technology

No. 1 Dai Co Viet, Hai Ba Trung District, Hanoi, Vietnam

Email: nam.nguyenhoang@ hust.edu.vn

\section{INTRODUCTION}

This paper is an extension of work originally presented in the 2019 International Conference on Advanced Computing and Applications (ACOMP) [1]. Electricity generation plays a significant role in increasing greenhouse gas emissions, and this situation is getting worse. In the US, electricity consumption in 2018 was 16 times higher than that in 1950 [2]. Also, in this year, electric energy primarily came from three sources: residential (39\%), commercial (36\%), and industry (25\%). It is clear that the highest proportion is in the residential section, so it's needed to take measures to reduce electricity consumption from this source.

According to [3], Sarah Darby estimated that could save up to $15 \%$ electrical energy if aware of the feedback on the power consumption of electrical appliances. Similarly, Sébastien Houde researched the effects of power consumption feedback on users on a large scale (1065 apartments in eight months) [4]. The results show that total power consumption reduced by about 5.7\%. Furthermore, Carrie Armela pointed out that if users were given feedback on the energy consumption of each electrical appliance, they could consume $12 \%$ less electrical energy [5].

Instead of changing users' habits, Tsai et al. built a system to automatically monitor and adjust indoor electricity usage using machine learning algorithms [6]. Research shows that electrical appliances in idle or standby mode account for 3-11\% of total indoor energy consumption. Therefore, the team trained the system to completely turn off electrical devices when not in use instead of operating in idle or standby mode. To accomplish this, many smart plugs with electrical metering and on/off functions are installed in the house.

Since it is time-consuming and costly to integrate each smart plug into every device, in this paper, the authors move towards changing users' habits by providing feedback on power consumption levels. To 
accomplish this purpose, the authors introduced a load monitoring device (LMD) to monitor the on/off status and power consumption of each electrical device in the house. Besides, an activity monitoring device (AMD) with an audio sensor is also used for the purpose of supporting LMD to identify electrical devices with the same power or continuously varying power over time.

\section{SURVEY ON MONITORING ELECTRICAL APPLIANCES}

Hart introduced the concept of non-intrusive appliance load monitoring (NALM) to indicate a system that identifies electrical appliances using only a single electronic meter [7]. Each electrical device has different active power $(\mathrm{P})$ and reactive power $(\mathrm{Q})$. Therefore, these two parameters are used as "Signature" for each electrical appliance.

Applying hart's device identification method, Weiss et al. used a commercial digital meter and software on the phone to perform the device recognition algorithms [8]. The algorithms allow us to add a new device through the software on the phone and then save the parameters of this device in a database. The system has a recognition accuracy of up to $87 \%$. Laughman et al. used harmonics as an identifier for each device [9]. The authors show that a computer and an incandescent bulb and have similar P and Q. However, only the computer can produce a third harmonic, and the incandescent lamp does not. Therefore, harmonics can be used to differentiate these two devices.

Another method developed by Norford and Leeb is analyzing the transient state [10]. Each device with a different structure will have a different switching power at start-up. Srinivasan et al. used harmonic parameters as inputs to various machine learning algorithms for electrical device identification [11]. The results showed that the machine learning method multilayer perceptron (MLP) and radial basis function (RBF) gave similar results and were better than the support vector machine (SVM) method. Patel et al. observed noise occurring on the line every time a device was turned on/off or operating [12]. The authors point out that the resistive load does not cause noise in operation but does cause transient noise when turned on or off. Inductive and solid-state loads cause additional noise during operation.

Lam et al. proposed a solution to use the voltage-current trajectory (V-I trajectory) as identifiers for each electrical appliance [13]. This method plots the graphs of voltage and current over one cycle and then relies on the shapes of graphs to analyze and classify electrical devices. Applying the V-I trajectory method in practice, Baets et al. used convolutional neural networks to analyze V-I trajectory images [14].

Because the application of machine learning algorithms in NALM is very potential, some papers evaluated the performance of some machine learning models in the NALM application [11], [15]. Kolter and Matthew have built a massive database for the development of identification algorithms [16]. The database includes information about the energy consumption of many devices in 10 homes for 19 days, with a total data capacity of up to 1 terabyte of raw data. In contrast, to reduce manual labeling data, Khaled Chahine developed a system that can extract the signatures and label them automatically [17].

Due to the hardware and software complexity in the NALM application, Semwal and Prasa focused on optimization algorithms using minimum features from smart meters [18]. Iksan et al. proposed a smoothing method for filtering out peak signals [19]. The system achieved better accuracy with this method.

Instead of using electric meters, Laput et al. used only a printed circuit board (PCB) with multiple sensors [20]. This board can recognize not only electrical devices but also indoor activities such as opening/closing doors, removing tissue paper, and draining the faucet. In particular, most events have a significant impact on the microphone and the accelerometer. The test was conducted in many places and achieved an accuracy of $96 \%$.

A lot of research about device state recognition already published. Those researches mainly focus on new identifiers to solve problems that exist when using old identifiers. In this paper, common electrical parameters and the MLP network model are used to identify the states of electrical appliances. Besides, the AMD with a microphone is also used for the purpose of supporting the identification of electrical devices with the same power or continuous varying power over time.

\section{THE SCOPE AND NOVELTY OF THE PAPER}

The authors conducted the experiment in a private house instead of a large building. We do not perform the identification of electrical appliances whose powers change continuously over time. The AMD with a microphone is capable of analyzing acoustic noises from running appliances to differentiate them. This device has been built to support LMD to realize time-varying power as well as similar power appliances. However, in this paper, we have not developed algorithms to combine data of LMD with AMD.

With the purpose of developing an accessible, easy-to-use, and low-cost measurement device, we have built the LMD based on open-source platforms (both hardware and software). The hardware schematics are relatively simple, with only voltage and current measurement channels. The Arduino software library is 
extremely accessible to newcomers. Therefore, it is much less time-consuming for newcomers to be familiar with the device. Also, they can easily customize the hardware and develop new features for the device.

As opposed to other mentioned papers in section 2, when the authors made many efforts to figure out new identifying characteristics from appliances, we use only some basic electrical parameters (e.g., voltage, current, and power) that can be obtained with the simple hardware to recognize household appliances. As demonstrated in [20], most events have an impact significantly on sound and vibration sensors. Thus, instead of using advanced electrical parameters (e.g., harmonics, noises on the line) that require complicated hardware, we only add the AMD with the microphone to support LMD to recognize appliances.

\section{PROPOSED SYSTEM DESIGN}

\subsection{Open-source design concepts}

The concept of open-source hardware was first released by Bruce Perens in 1997. Open-source hardware makes it easy for everyone to design and develop new features for their own projects. In addition, open-source software is said to be highly reliable because many communities are involved in debugging and testing. As a result, open-source will make it faster and easier for people to conduct their projects. Thanks to these reasons, open-source boards are also of great use for education [21], [22].

Arduino is a company that specializes in providing boards with open source in both hardware and software. Arduino provides an easy-to-use integrated development environment (IDE) that contains many simple functions and libraries in $\mathrm{C}++$ languages. In this paper, in order to have an easy-to-customize measuring device, we developed a digital meter, LMD, based on the Arduino Due board. Similarly, a popular open hardware device is Shenzhen Xunlong Software's Orange Pi Zero board. This board uses a highperformance $\mathrm{ARM}{ }^{\circledR}$ Cortex $^{\mathrm{TM}}-\mathrm{A} 7$ microprocessor, so $\mathrm{AMD}$ uses this board to analyze sound and differentiate home electrical appliances.

\subsection{System architecture}

As shown in Figure 1, there are two types of monitoring devices: LMD and AMD. The LMD is programmed to measure electrical parameters with a cycle of one second. LMD passes these data to a computer to run machine learning algorithms that predict the state of electrical appliances. In the following sections of the paper, the authors will analyze problems in using LMD to identify electrical appliances. The first problem is to distinguish devices with similar power consumption, and the second is to distinguish devices with varying power over time. Therefore, AMD equipment with the audio sensor is used to overcome these problems in the future.

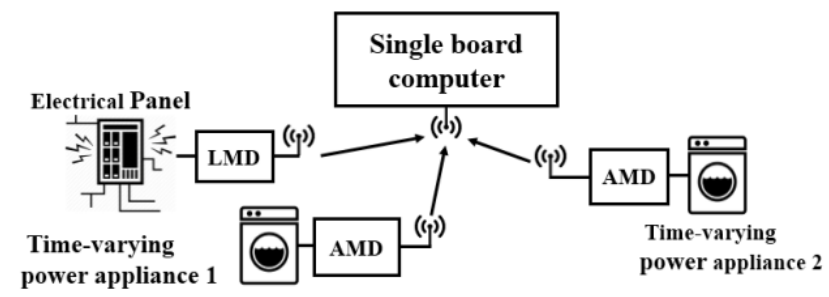

Figure 1. The proposed system model

\section{LOAD MONITORING DEVICE (LMD)}

\subsection{Hardware structure and measurement program}

Figure 2 shows the block diagram of the LMD. Because the LMD is integrated into the electrical panel, LMD are powered by the 220 VAC grid. The LMD measurement circuit includes the voltage and current measuring channel. The schematics of these measuring channels are built based on the reference design of microchip technology [23], [24]. Similarly, as mentioned above, the Arduino Due board is used as the central processing unit of the LMD. This block will process the signals from the voltage and current channels, display the calculated parameters on the linear complementary dual (LCD), send them to the computer via $\mathrm{Wi}-\mathrm{Fi}$, and store the energy consumed on the electrically erasable programmable read-only memory (EEPROM). LMD can measure six electrical parameters, which are voltage (Urms), current (Irms), active power $(\mathrm{P})$, reactive power $(\mathrm{Q})$, power factor $(\cos \varphi)$, and energy consumption $(\mathrm{E})$. Urms and Irms are calculated by (1), as shown in Figure 2. 


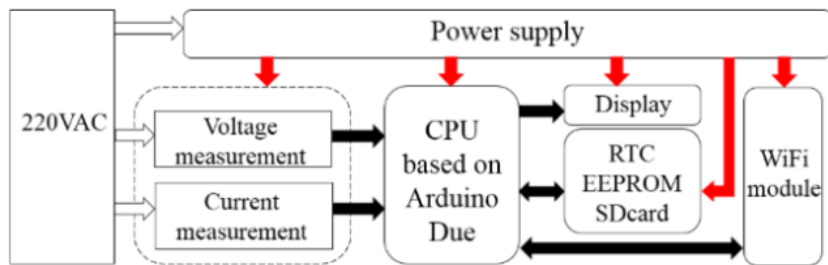

Figure 2. The hardware block diagram of LMD

$$
U_{r m s}=\sqrt{\frac{\sum_{n=0}^{N-1} u^{2}(n)}{N}} ; I_{r m s}=\sqrt{\frac{\sum_{n=0}^{N-1} i^{2}(n)}{N}}
$$

Active power and reactive power are calculated from $u(n)$ and $i(n)$, as shown in (2). Where $u(n)$, $i(n)$, and $i_{90-\text { degree-shift }}(n)$ are instantaneous voltage, instantaneous current, and instantaneous current shifted by 90 degrees. $N$ is the number of samples. The sampling frequency is $2000 \mathrm{~Hz}$, the measurement data update rate is $1 \mathrm{~Hz}$, so $N=2000$.

$$
P=\frac{\sum_{n=1}^{N}[u(n) \times i(n)]}{N} ; Q=\frac{\sum_{n=1}^{N}\left[u(n) \times i_{90-\text { deg ree-shift }}(n)\right]}{N}
$$

\subsection{Appliance states detecting algorithm}

In recent years, artificial intelligent (AI) has become a phenomenon in the world. Machine learning (ML) is a subset of AI. Machine learning is traditionally divided into three main groups: supervised learning, unsupervised learning, and reinforcement learning. In this system, we combine the supervised learning method with a three-layer MLP to train the system in realizing the state of electrical appliances. As shown in Figure 3 and (3), the input, hidden, and output layers are represented by vectors $x, a$, and $y$, respectively. Matrix weight $W$ and bias $b$ represent connections between the two layers.

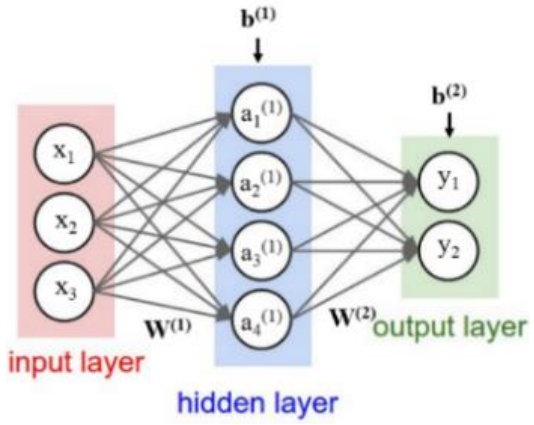

Figure 3. Three layers multilayer perceptron (MLP)

$$
x=\left[\begin{array}{c}
x_{1} \\
x_{2} \\
\ldots
\end{array}\right] ; a^{(l)}=\left[\begin{array}{c}
a_{1}^{(l)} \\
a_{2}^{(l)} \\
\ldots
\end{array}\right] ; y=\left[\begin{array}{c}
y_{1} \\
y_{2} \\
\ldots
\end{array}\right] ; W=\left[\begin{array}{ccc}
w_{11}^{(l)} & w_{12}^{(l)} & \ldots \\
w_{21}^{(l)} & w_{22}^{(l)} & \ldots \\
\ldots & \ldots & w_{i j}^{(l)}
\end{array}\right] ; b=\left[\begin{array}{c}
b_{1}^{(l)} \\
b_{2}^{(l)} \\
\ldots
\end{array}\right]
$$

As demonstrated in (4) describe the relationship between the input and output layer. Where $l$ is layer number, $l=1,2, \ldots, \mathrm{L}$ (L is the last layer, $a^{(0)}$ is input vector, $a^{(\mathrm{L})}$ is output vector), $g(z)$ is activation function which is sigmoid function shown in (5).

$$
\begin{aligned}
& z^{(l)}=W^{(l)} a^{(l-1)}+b^{(l)} ; a^{(l)}=g\left(z^{(l)}\right) \\
& g(z)=\frac{1}{1+e^{-z}}
\end{aligned}
$$


First, we must determine the components of the input vector $\boldsymbol{x}$ and the output vector $\boldsymbol{y}$. In this case, the input vector is the electrical parameters obtained from the LMD. The output vector is the on/off states of each device. The next step is to determine the optimum $W$ and $b$ matrices to show the relationship between the input and output vectors. With the supervised training method, a training data set is used to find these two matrices. The test data set is then used to test the predictive accuracy of the system.

Active power P and reactive power Q are used as "Signature" for each device. Table 1 shows the power of some indoor appliances to be predicted in the experiment section. We can see from the table that the devices have different $\mathrm{P}$ and $\mathrm{Q}$.

Observing the load graph in Figure 4, each time a device is turned on or off, a rising or falling edge appears on the graph. The amplitude of the edge is the power consumption of the device that has been turned on/off. We developed an algorithm called "edge detection" to identify the potential edges. Even when no appliances are turned on/off, the total power is constantly changing. Thus, it is necessary to define a threshold value large enough for the algorithm to eliminate these fluctuations. In this paper, the oscillation threshold for $\mathrm{P}$ and $\mathrm{Q}$ are $15 \mathrm{~W}$ and $8 \mathrm{VAr}$, respectively.

Table 1. Specifications of some electrical devices used for the experiment

\begin{tabular}{ccccc}
\hline No & Appliance & Real Power $(\mathrm{W})$ & Reactive Power (VAr) & Power Factor $(\cos \varphi)$ \\
\hline 1 & Hairdryer (mode 1) & 455 & 13 & 0.99 \\
2 & Hairdryer (mode 2) & 893 & 31 & 0.99 \\
3 & Kettle 1 & 1374 & 5 & 0.99 \\
4 & Kettle 2 & 1958 & 50 & 0.99 \\
5 & LED lamp & 62 & 11 & 0.89 \\
6 & Compact lamp & 45 & -8 & 0.99 \\
7 & Fan (with electronic circuit) & 60 & -12 & 0.96 \\
8 & Incandescent lamp & 202 & 0 & 1.0 \\
9 & Chandelier & 260 & 0 & 1.0 \\
10 & Heating lamp (mode 1) & 526 & 0 & 1.0 \\
11 & Heating lamp (mode 2) & 30 & 0 & 1.0 \\
12 & Fluorescent lamp & 730 & 69 & 0.4 \\
13 & Heating bag & & 0 & 1.0 \\
\hline
\end{tabular}

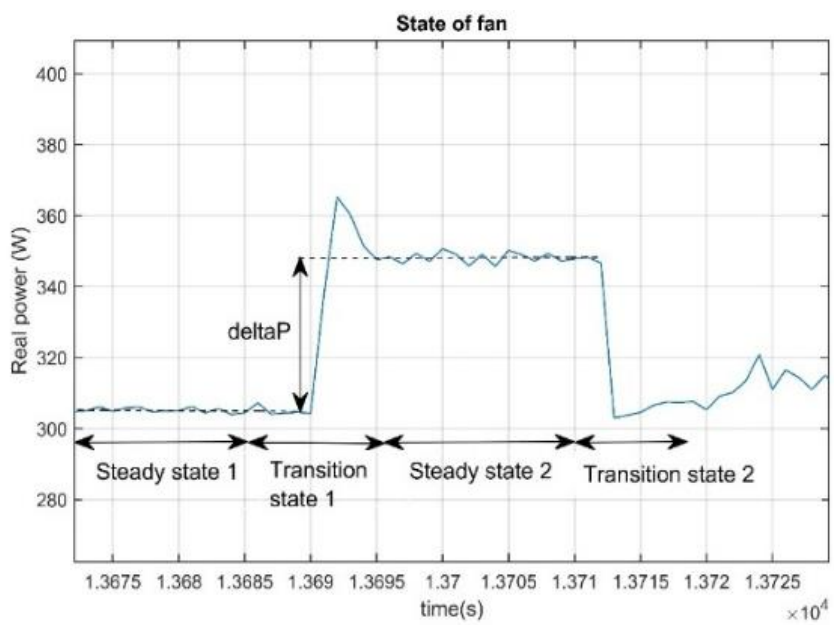

Figure 4. Rising and falling edge when turning on/off the fan

We have the following input vector of the MLP network:

$$
x=\left[\begin{array}{l}
\Delta P \\
\Delta Q
\end{array}\right]
$$

A total of 12 devices are used during the test, in which hair-dryer and heating lamp devices have multiple operating modes. Thus, the 32 outcomes are represented by vectors, as shown in Tables 2 and 3 . As shown in (7) specifically describes a pair of input vectors $x^{(1)}$ and output vectors $y^{(1)}$. Where $m$ is the number of data points collected, the matrices $X$ and $Y$ are the product of combining all $m$ input and output vectors 
$\left(\mathrm{X} \in \mathbb{R}^{2 \times m}, \mathrm{Y} \in \mathbb{R}^{32 \times m}\right)$. These data are divided into two groups as the training set and test set. Training set is used to train the MLP model. The Test Set is used to evaluate the prediction accuracy of the MLP model after it has been trained. The goal of the training process is to find two optimal matrices $W$ and $b$, so that the predicted outputs approximate the actual outputs. The difference between these two outputs is evaluated using (8) (the error function).

Table 2. Output vector $\mathbf{y}$

\begin{tabular}{cccccc}
\hline Output & 1 & 2 & 3 & $\ldots$ & 32 \\
\hline $\boldsymbol{y}$ (Output vector) & {$\left[\begin{array}{c}1 \\
0 \\
0 \\
\cdots \\
0\end{array}\right]\left[\begin{array}{c}0 \\
1 \\
0 \\
\cdots \\
0\end{array}\right]\left[\begin{array}{c}0 \\
0 \\
1 \\
\cdots \\
0\end{array}\right]$} & & & & \\
& & & {$\left[\begin{array}{c}0 \\
0 \\
0 \\
\cdots \\
1\end{array}\right]$} \\
\hline
\end{tabular}

Table 3. Output vector and device states

\begin{tabular}{cc}
\hline Output & State \\
\hline 1 & Hairdryer is on (mode 1) \\
2 & Hairdryer is off (mode 1) \\
$\ldots$ & $\ldots$ \\
32 & Heating bag is off \\
\hline
\end{tabular}

$$
\begin{aligned}
& x^{(1)}=\left[\begin{array}{l}
455.36 \\
12.19
\end{array}\right] ; y^{(1)}=\left[\begin{array}{l}
1 \\
0 \\
\ldots \\
0
\end{array}\right] ; X=\left[\begin{array}{ccc}
\mid & \mid & \ldots \\
x^{(1)} & x^{(2)} & \ldots \\
\mid & \mid & \ldots
\end{array}\right] ; Y=\left[\begin{array}{ccc}
\mid & \mid & \ldots \\
y^{(1)} & y^{(2)} & \ldots \\
\mid & \mid & \ldots
\end{array}\right] \\
& J=\frac{1}{m} \sum_{i=1}^{m} \sum_{k=1}^{K}-y_{k}{ }^{(i)} \log _{e} a_{k}^{L(i)}-\left(1-y_{k}^{(i)}\right) \log _{e}\left(1-a_{k}^{L(i)}\right)
\end{aligned}
$$

Where $a_{k}{ }^{L}$ is predicted output $k$ of the output layer, $y_{k}$ is the corresponding label $k$ in Training Set, $K$ is the number of units of the output layer $(K=32), m$ is the total number of collected data in Training Set. With cost function $\mathbf{J}$, the value $\mathbf{J}$ is small when $a_{k}{ }^{L} \approx y_{k}$. The problem to be solved now is to find the minimum value of the function $\mathrm{J}$; from there, we have the matrices $\boldsymbol{W}$ and $\boldsymbol{b}$, respectively. Finding the minimum value of $\mathrm{J}$ by solving as shown in (8) is a complicated task, and thus Gradient Descent and Backward propagation are two useful algorithms to solve this problem [25].

Overall, the process of recognizing the on/off states of electrical devices is described in Figure 5. The computer will always collect the electrical parameters from the meter over Wi-Fi. Once all the parameters have been received, the computer runs the "edge detection" algorithm to detect the changes of $\mathrm{P}$ and $\mathrm{Q}$. If it detects an edge $\Delta \mathrm{P}$ and $\Delta \mathrm{Q}$, the computer runs the machine learning algorithm to identify which appliance has just been turned on or off. After that, the computer will return to collect new data from the meter.

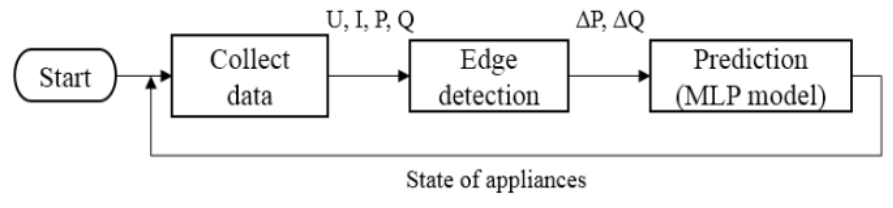

Figure 5. Process of identifying electrical appliance state

\section{ACTIVITY MONITORING DEVICE (AMD)}

The disadvantage when identifying devices from power $\mathrm{P}$ and $\mathrm{Q}$ is that it is challenging to identify devices with the same or continuously varying powers over time. AMD equipment is developed for smallscale installations solely to address the two problems mentioned above. According to [20], the two sensors with the most data change every time an event occurs: the sound sensor and the accelerometer. Therefore, the authors use a microphone for AMD to implement system support for device recognition. In this paper, the authors only test AMD functionality but have not combined LMD to identify appliances.

The hardware structure of AMD is illustrated in Figure 6. Direct current (DC) power is converted from a $220 \mathrm{~V}$ alternating current (AC) power line to supply the Orange Pi Zero board, a microphone, and a display. Orange Pi Zero runs Ubuntu Ambian operating system and supports Python language. We deploy Python's libraries related to the fast fourier transform (FFT) algorithm for analyzing sounds from electrical appliances. 


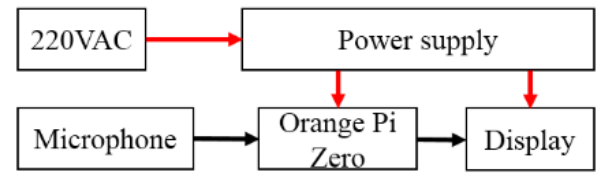

Figure 6. The hardware block diagram of AMD

We performed an acoustic analysis from three typical electrical appliances, the hairdryer, the fan, and the microwave oven. Fan noise mainly comes from ball bearings and propellers when rotating. The sound from the hairdryer is generated by the heater, front and rear grids, and the air filter. The microwave noise comes from the cooling fan to make sure the magnetron does not heat up. Figure 7 comparing measurement results in the spectrum of Figure 7 (a) fan $(57-58 \mathrm{~Hz})$ and Figure 7 (b) hairdryer $(900-940 \mathrm{~Hz})$ after ten times of recording and analyzing the sound of three devices at a distance of 1 meter. Figure 8 comparing measurement results in the spectrum of Figure 8(a) microwave oven $(195-210 \mathrm{~Hz})$ and Figure 8(b) all three appliances at the same time. It is clear that each device's feature still appears clearly when all of the three appliances operate simultaneously. Therefore, this feature can be used as the "Signature" for each device.

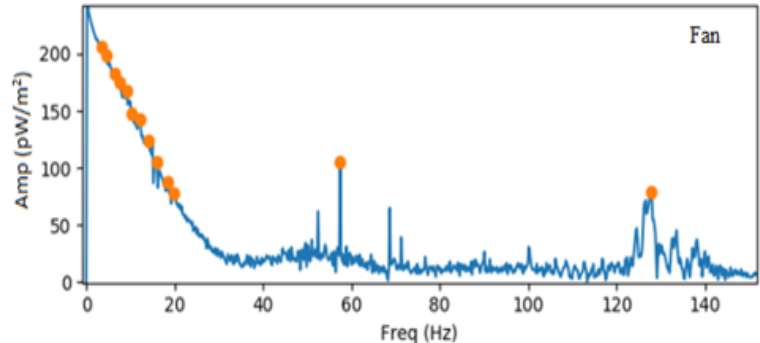

(a)

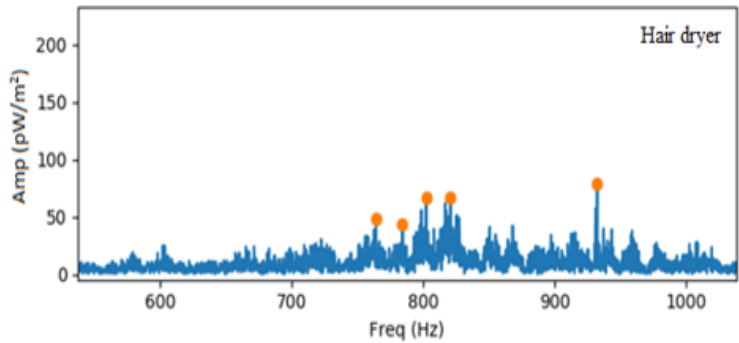

(b)

Figure 7. Spectrums of (a) fan VINAWIND QB 300Đ and (b) hairdryer PHILIPS HP 4840

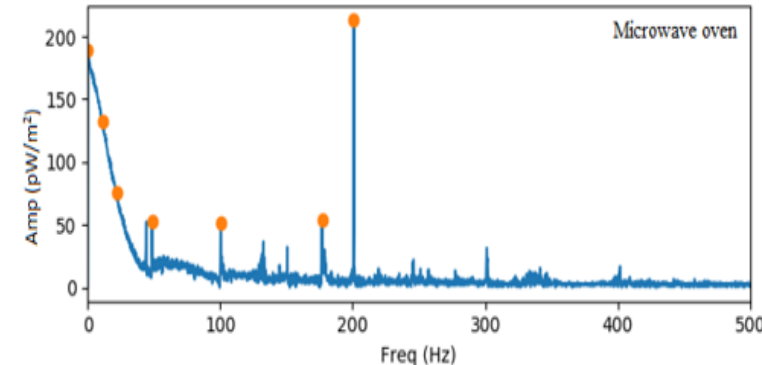

(a)

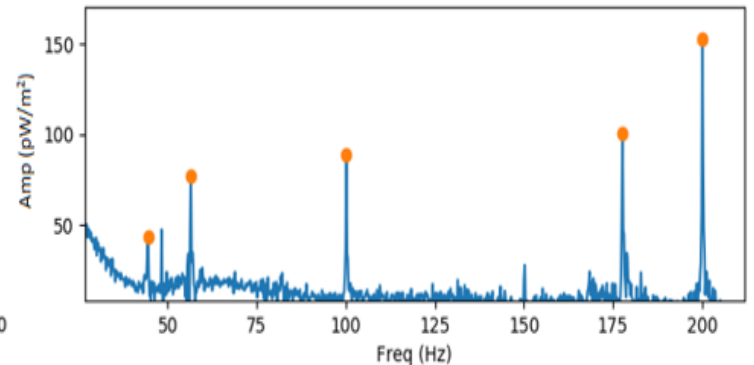

(b)

Figure 8. Spectrums of (a) microwave oven DAEWOO KOG-1A4H and (b) three appliances

\section{EXPERIMENTS}

Figure 9 comparing device PCBs of Figure 9(a) the LMD board and Figure 9(b) AMD board. The two boards are based on open-source designs from Microchip/Arduino and the Orange Pi Zero. LMD can measure Urms, Irms, active power P, reactive power Q, power factor, and energy consumption E. The computer will receive these parameters from the LMD via Wi-Fi to perform the algorithm in Figure 5. The accuracy of both voltage and current channels is under $1 \%$ after being calibrated.

The authors tested the monitoring system at the private house, including the bathroom, laundry room, and bedroom. The current transformer of the electronic meter was installed to measure power simultaneously in three rooms. The number of devices is listed in Table 1; some of them have multiple operating modes such as hairdryer and heating lamp, so a total of 32 on/off cases needs to be recognized. The 
experiment was performed on weekday evenings (monday to friday). At the end of the week, the authors ran the test all day. The total duration of the experiment was three weeks. The MLP model was trained to recognize $32 \mathrm{on} /$ off cases before conducting the experiment. A total of 215 data points were collected. The $70 \%$ points for the Training set and $30 \%$ points for the Test set. After the end of 30000 iterations, the value of the error function $\mathrm{J}$ is 0.295 . The prediction result is $93.65 \%$ accurate on the test set. After three weeks, the system predicted a total of 766 events, of which 49 were wrongly predicted. As a result, the system achieved a prediction accuracy of $93.60 \%$. Figure 10 shows the active and reactive power graph during the $4^{\text {th }}$ test day. After testing, the authors found some disadvantages of the current algorithm. Figure 11 comparing measurement power of (a) warmer bag and (b) washing machine. The algorithms based on $\mathrm{P}$ and $\mathrm{Q}$ perform poorly for devices with continuously varying power. For example, observe Figure 11(a), the power of the heating bag gradually increases by more than $240 \mathrm{~W}$ over time. Likewise, the power of the washing machine changes continuously between $1958 \mathrm{~W}$ and $2150 \mathrm{~W}$ in soaking mode as shown in Figure 11(b). Therefore, the algorithm is unable to obtain the correct $\Delta \mathrm{P}$ value. Second, the system confuses devices with nearly the same power, such as incandescent and compact lamps (almost identical active power P). In some cases, the reactive power $\mathrm{Q}$ of the compact lamp is less than the detection threshold of the edge detection algorithm of 8 VAr. The algorithm ignores this power edge, thus incorrectly predicting the incandescent lamp. We built AMD with the microphone to tackle the above two problems in the future. The first version of AMD using the Orange Pi Zero board. The three devices used in the recognition experiment are the hairdryer, the fan, and the microwave oven. The test was conducted at a distance from 0.5 to $2 \mathrm{~m}$. At each distance, AMD predicts the device state multiple times to evaluate prediction accuracy. Table 4 shows the test results; we can see that the system is most accurate at a distance of less than $1.5 \mathrm{~m}$.

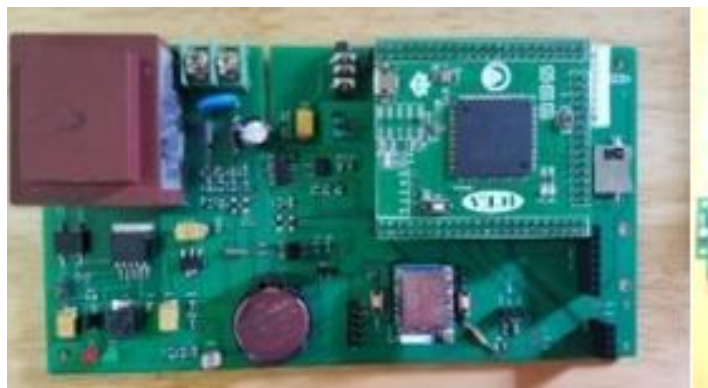

(a)

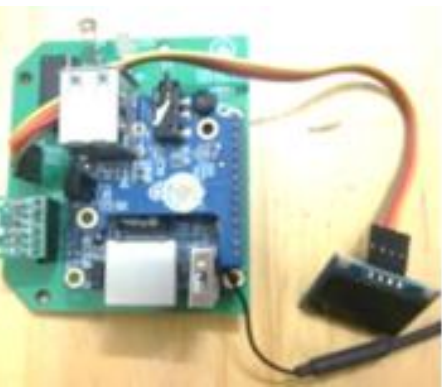

(b)

Figure 9. Designing of the (a) LMD board and (b) AMD board
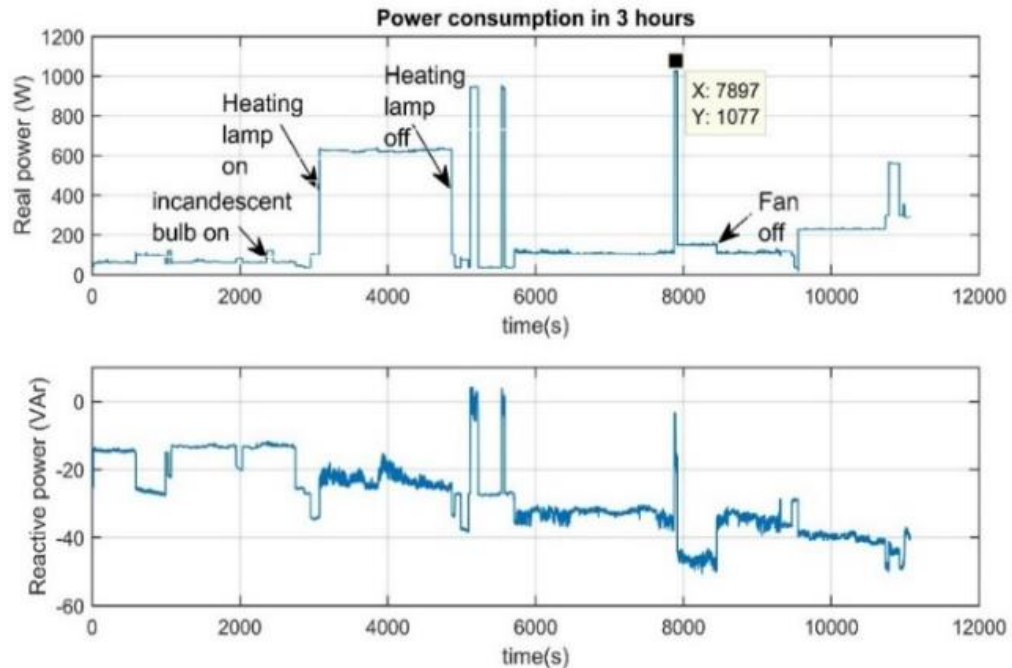

Figure 10. Power consumption graph in $4^{\text {th }}$ test day 


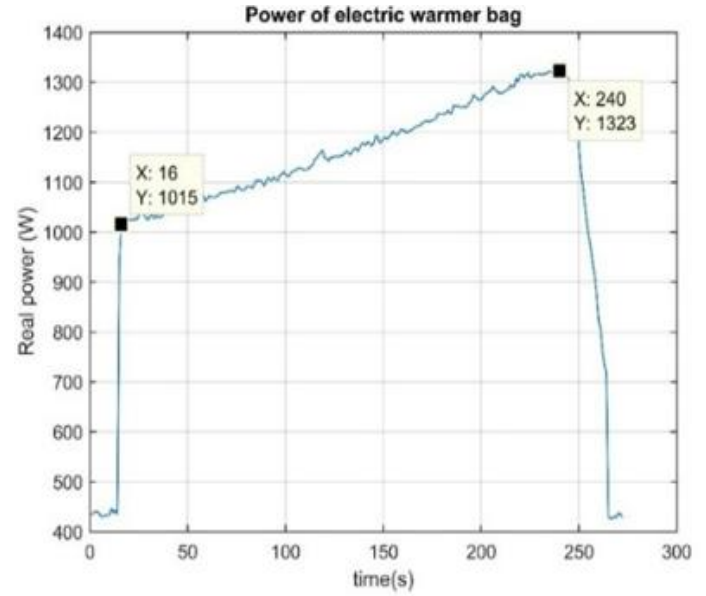

(a)

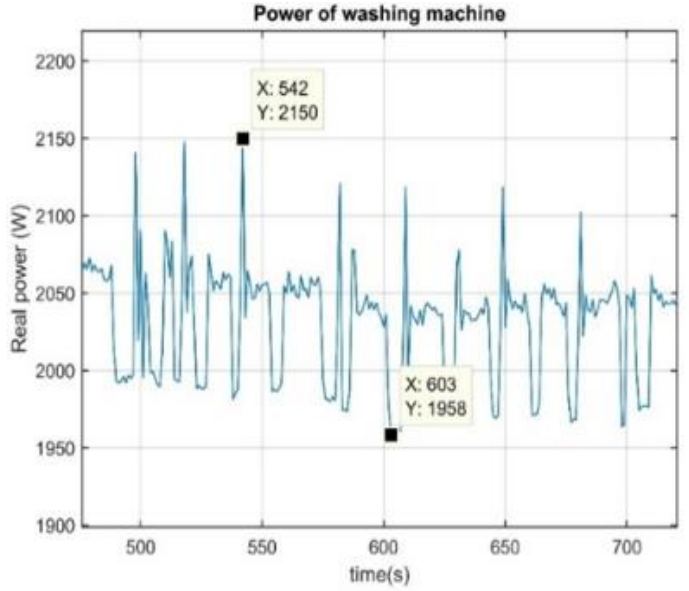

(b)

Figure 11. Power of (a) warmer bag and (b) washing machine

Table 4. AMD test results

\begin{tabular}{ccccc}
\hline & $0.5 \mathrm{~m}$ & $1 \mathrm{~m}$ & $1.5 \mathrm{~m}$ & $2 \mathrm{~m}$ \\
\hline Fan & $40 / 40(100 \%)$ & $40 / 40(100 \%)$ & $35 / 40(88 \%)$ & $19 / 40(48 \%)$ \\
Hair Dryer & $40 / 40(100 \%)$ & $39 / 40(98 \%)$ & $37 / 40(93 \%)$ & $25 / 40(62.5 \%)$ \\
Microwave Oven & $20 / 20(100 \%)$ & $20 / 20(100 \%)$ & $19 / 20(95 \%)$ & $13 / 20(65 \%)$ \\
\hline
\end{tabular}

\section{CONCLUSION}

This paper presents two devices call LMD and AMD. LMD is the electronic meter that is installed at the electrical panel of a room or a house. LMD provides information about power consumption in order to detect which electrical appliances are running. This device provides electricity consumption information of each electrical appliance to homeowners so that they can adjust their usage plans more efficiently. Meanwhile, AMD is equipped with the microphone; the function of this device is to provide more information on appliances with time-varying power or similar power. The authors have built the first version of the electrical equipment state recognition system using an open-source platform. The system applies the supervised learning method and the MLP model. Active power P and reactive power Q are used as Signatures for each device. The experiment was conducted over three weeks in three different rooms. The authors trained the system to identify 12 devices in which two devices have multiple modes. The accuracy of the system is $93.60 \%$. However, during testing, the authors found that the system works inefficiently for devices with constantly changing power. It is also difficult for the system to differentiate devices with similar power. Therefore, the authors intend to combine data from AMD to solve the two analyzed problems in the future.

\section{REFERENCES}

[1] N. H. Nguyen and V. H. Duong, "A system for monitoring the electric usage of home appliances using machine learning algorithms," in Proceedings - 2019 International Conference on Advanced Computing and Applications, ACOMP 2019, Nov. 2019, pp. 158-164, doi: 10.1109/ACOMP.2019.00032.

[2] "Electricity explained-use of electricity," U.S. Energy Information Administration, 2020. https://www.eia.gov/energyexplained/electricity/use-of-electricity.php (accessed Feb. 2020).

[3] S. Darby, "The Effectiveness of feedback on energy consumption," Environmental Change Institute University of Oxford, pp. 121, 2006, [Online]. Available: https://www.eci.ox.ac.uk/research/energy/downloads/smart-metering-report.pdf.

[4] S. Houde, A. Todd, A. Sudarshan, J. A. Flora, and K. C. Armel, "Real-time feedback and electricity consumption: A field experiment assessing the potential for savings and persistence," Energy Journal, vol. 34, no. 1, pp. 87-102, Jan. 2013, doi: 10.5547/01956574.34.1.4.

[5] K. Carrie Armel, A. Gupta, G. Shrimali, and A. Albert, "Is disaggregation the holy grail of energy efficiency? The case of electricity," Energy Policy, vol. 52, pp. 213-234, Jan. 2013, doi: 10.1016/j.enpol.2012.08.062.

[6] K. L. Tsai, F. Y. Leu, and I. You, "Residence energy control system based on wireless smart socket and IoT," IEEE Access, vol. 4, pp. 2885-2894, 2016, doi: 10.1109/ACCESS.2016.2574199.

[7] G. W. Hart, "Nonintrusive appliance load monitoring," Proceedings of the IEEE, vol. 80, no. 12, pp. 1870-1891, 1992, doi: $10.1109 / 5.192069$

[8] M. Weiss, A. Helfenstein, F. Mattern, and T. Staake, "Leveraging smart meter data to recognize home appliances," in 2012 IEEE International Conference on Pervasive Computing and Communications, PerCom 2012, Mar. 2012, pp. 190-197, doi: 10.1109/PerCom.2012.6199866.

[9] C. Laughman et al., "Power signature analysis," IEEE Power and Energy Magazine, vol. 1, no. 2, pp. 56-63, Mar. 2003, doi:

Int J Artif Intell, Vol. 11, No. 1, March 2022: 300-309 
10.1109/MPAE.2003.1192027.

[10] L. K. Norford and S. B. Leeb, "Non-intrusive electrical load monitoring in commercial buildings based on steady-state and transient load-detection algorithms," Energy and Buildings, vol. 24, no. 1, pp. 51-64, Jan. 1996, doi: 10.1016/03787788(95)00958-2.

[11] D. Srinivasan, W. S. Ng, and A. C. Liew, "Neural-network-based signature recognition for harmonic source identification," IEEE Transactions on Power Delivery, vol. 21, no. 1, pp. 398-405, Jan. 2006, doi: 10.1109/TPWRD.2005.852370.

[12] S. N. Patel, T. Robertson, J. A. Kientz, M. S. Reynolds, and G. D. Abowd, "At the flick of a switch: detecting and classifying unique electrical events on the residential power line," in UbiComp 2007: Ubiquitous Computing, Springer Berlin Heidelberg, 2007, pp. 271-288.

[13] H. Y. Lam, G. S. K. Fung, and W. K. Lee, "A novel method to construct taxonomy electrical appliances based on load signatures," IEEE Transactions on Consumer Electronics, vol. 53, no. 2, pp. 653-660, 2007, doi: 10.1109/TCE.2007.381742.

[14] L. De Baets, J. Ruyssinck, C. Develder, T. Dhaene, and D. Deschrijver, "Appliance classification using VI trajectories and convolutional neural networks," Energy and Buildings, vol. 158, pp. 32-36, Jan. 2018, doi: 10.1016/j.enbuild.2017.09.087.

[15] K. Khalid, A. Mohamed, R. Mohamed, and H. Shareef, "Performance comparison of artificial intelligence techniques for nonintrusive electrical load monitoring," Bulletin of Electrical Engineering and Informatics, vol. 7, no. 2, pp. 143-152, Jun. 2018, doi: $10.11591 /$ eei.v7i2.1190

[16] J. Z. Kolter and M. J. Johnson, "REDD : a public data set for energy disaggregation research," in SustKDD workshop, 2011, no. 1, pp. 1-6.

[17] K. Chahine, "Towards automatic setup of non intrusive appliance load monitoring - Feature extraction and clustering," International Journal of Electrical and Computer Engineering, vol. 9, no. 2, pp. 1002-1011, Apr. 2019, doi: 10.11591/ijece.v9i2.pp1002-1011.

[18] S. Semwal, R. S. Prasad, and P. Juneja, "Identifying appliances using NIALM with minimum features," International Journal of Electrical and Computer Engineering, vol. 4, no. 6, pp. 909-922, Dec. 2014, doi: 10.11591/ijece.v4i6.6715.

[19] N. Iksan, J. Sembiring, N. Hariyanto, and S. H. Supangkat, "Residential load event detection in NILM using robust cepstrum smoothing based method," International Journal of Electrical and Computer Engineering, vol. 9, no. 2, pp. 742-752, Apr. 2019, doi: 10.11591/ijece.v9i2.pp742-752.

[20] G. Laput, Y. Zhang, and C. Harrison, "Synthetic sensors: towards general-purpose sensing," in Conference on Human Factors in Computing Systems - Proceedings, May 2017, vol. 2017-May, pp. 3986-3999, doi: 10.1145/3025453.3025773.

[21] J. Jalden, X. C. Moreno, and I. Skog, "Using the arduino due for teaching digital signal processing," in ICASSP, IEEE International Conference on Acoustics, Speech and Signal Processing - Proceedings, Apr. 2018, vol. 2018-April, pp. 6468-6472, doi: 10.1109/ICASSP.2018.8461781.

[22] C. Hochgraf, "Using arduino to teach digital signal processing," 2013, [Online]. Available: https://pdfs.semanticscholar.org/290a/8c9aab485d3b8f4ebe8e08584bd300d7666c.pdf.

[23] "Atmel AVR465: Single-Phase Power/Energy Meter with Tamper Detection," 2013. [Online]. Available: http://ww1.microchip.com/downloads/en/Appnotes/Atmel-2566-Single-Phase-Power-Energy-Meter-with-Tamper-Detection_ApNotes_AVR465.pdf.

[24] "Atmel AVR1631: Single Phase Energy Meter using XMEGA A," 2012. [Online]. Available: http://ww1.microchip.com/downloads/en/Appnotes/doc42039.pdf.

[25] T. H. Vu, The fundamentals of Machine Learning. 2018.

\section{BIOGRAPHIES OF AUTHORS}

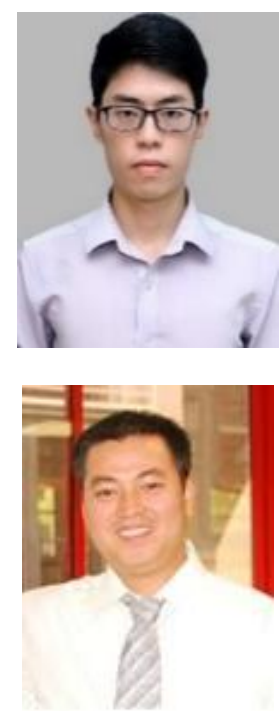

Viet Hoang Duong (D) BD SC P received his bachelor's degree in 2016 and Master's degree in 2020 from Hanoi University of Science and Technology (HUST), Vietnam. His research interests include smart grids, machine learning, and instrumentation. He can be contacted at email: viet.dhca180160@ sis.hust.edu.vn.

Nam Hoang Nguyen (D) 8f SC P received his engineer degree in 2002 from Hanoi University of Technology (HUST), his Master's degree in 2004 from Hendri Poincaré University, France, and his Ph.D. in 2009 from Grenoble Polytechnic University, France. He is currently a Lecturer in the Department of Industrial Metrology and Informatics (3I), School of Electrical Engineering, Hanoi University of Technology (HUST). His main research directions are intelligent metering systems, IoT and embedded systems, IIoT, and renewable energy systems. He can be contacted at email: nam.nguyenhoang@ hust.edu.vn. 